\title{
COSTOS DE CUMPLIMIENTO Y PODER DE MERCADO: APLICACION AL PROGRAMA DE COMPENSACION DE EMISIONES*
}

\author{
Clara Inés Villegas** \\ Carlos Chávez ${ }^{* * *}$
}

\begin{abstract}
This article analyzes the impact of market power on a transferable emissions permit system (TEPS). The application is carried out for the case of the "Programa de Compensación de Emisiones" in the city of Santiago, Chile, using data for 1997. In addition to the abatement costs, the costs of enforcing the system are also considered. Specifically, the magnitude of the efficiency loss caused by a non-optimal initial allocation of permits is quantified. Our results show that the costs of inducing complete compliance are important and its relevance depends on the target level of the aggregate emissions. Finally, the results also suggest that the presence of market power causes a modest increase in total compliance costs. The result is robust to different levels for the parameters used in the simulations.

* Los autores agradecemos de manera especial a dos árbitros anónimos evaluadores de la revista Cuadernos de Economía por sus valiosos comentarios, que contribuyeron a aclarar y a mejorar la calidad del trabajo que aquí se presenta. De igual forma, expresamos nuestros agradecimientos a Jorge Dresdner y Mauricio Villena, por sus sugerencias al trabajo inicial fuente del presente artículo; adicionalmente, agradecemos a la Oficina de Calidad del Aire-SESMA por haber proporcionado la base de datos sobre la cual se realizó el trabajo empírico. Los errores que persistan son de nuestra exclusiva responsabilidad.

** Profesor Instructor, Escuela de Geociencias y Medio Ambiente, Facultad de MinasUniversidad Nacional de Colombia, Medellín, Colombia.

E-mail: civilleg@unalmed.edu.co

*** Profesor Asistente, Departamento de Economía, Universidad de Concepción.

E-mail: cchavez@udec.cl.

JEL classification: L51, Q52

Keywords: Transferable Emissions Permit, Market Power, Compliance Costs, Efficiency.
\end{abstract}




\section{RESUMEN}

Este artículo analiza desde un punto de vista empírico el impacto de la presencia de poder de mercado en un sistema de permisos de emisión transferibles (SPET). La aplicación se realiza para el caso del Programa de Compensación de Emisiones (PCE) de la ciudad de Santiago de Chile, utilizando datos del año 1997. El análisis de este artículo considera explícitamente, además de los costos de abatimiento de emisiones, los costos de fiscalización para inducir cumplimiento de la regulación. Específicamente, se cuantifica la magnitud de la pérdida de eficiencia causada por asignaciones iniciales de permisos no óptimas. Nuestros resultados muestran que los costos de inducir un completo cumplimiento son importantes y su relevancia depende de la meta ambiental deseada. Los resultados sugieren, además, que la presencia de poder de mercado produce un incremento modesto en los costos totales de cumplimiento. Este resultado es robusto para diferentes niveles de los parámetros usados en las simulaciones.

\section{INTRODUCCIÓN}

El control de la contaminación y el consecuente mejoramiento de la calidad ambiental puede ser logrado a través de diversas formas de regulación. El interés por el uso de instrumentos económicos como una alternativa para el mejoramiento de la calidad ambiental ha sido creciente, y se explica porque éstos ofrecen la posibilidad de dicho logro a costos menores que los ocasionados por alternativas de regulación tradicionales del tipo comando y control. Entre los instrumentos económicos disponibles para el control de la contaminación se destacan los Sistemas de Permisos de Emisión Transferibles (SPET). Dichos sistemas están basados y dependen críticamente de la creación y adecuado funcionamiento de un mercado.

La promesa teórica de mejorar la calidad ambiental de la manera más barata posible mediante la implementación de un SPET puede ser amenazada, entre otros, por dos problemas fundamentales. En primer lugar, la presencia de poder de mercado puede afectar el desempeño de un mercado de permisos de emisión transferibles. En efecto, en presencia de poder de mercado, la distribución final de niveles de emisión puede no coincidir con aquella distribución que minimiza los costos agregados de abatimiento. Adicionalmente, en presencia de poder de mercado la distribución de niveles de emisión depende de la asignación inicial de permisos, pasando a tener esta última no sólo implicancias de tipo distributivas sino también de eficiencia. Hahn (1984) fue el primero en mostrar que el resultado principal de Montgomery (1972) respecto al funcionamiento de un sistema de permisos transferibles puede no cumplirse cuando el mercado de permisos no es perfectamente competitivo. En presencia de poder de mercado, una firma participante (dominan- 
te) posee la capacidad de manipular el precio de los permisos a través de sus transacciones, alterándolo respecto de su nivel competitivo. Debido a que las decisiones de abatimiento de las restantes firmas participantes (competitivas) dependen del precio de los permisos, la manipulación del mismo puede ocasionar que éstas alteren dichas decisiones, influyendo directamente en los costos de cumplimiento de la regulación y, por tanto, en la característica de costo-efectividad de la política.

En segundo lugar, la realización práctica de las ganancias de eficiencia potenciales de un SPET depende de manera crítica de los niveles de cumplimiento logrados, los cuales a su vez están determinados por el proceso de fiscalización de parte de las autoridades. En el análisis teórico y empírico de políticas ambientales basadas en un SPET, se ha tendido a ignorar aspectos referidos a la necesidad de diseñar e implementar estrategias para inducir adecuados niveles de cumplimiento de parte de los agentes regulados. La implementación de tales actividades involucra costos para la agencia reguladora; por tanto, cuando se ignoran los requerimientos para inducir cumplimiento de la política, se tiende a subestimar los costos de cumplimiento de la regulación.

Este artículo analiza desde un punto de vista empírico el impacto de la presencia de poder de mercado en un sistema de permisos de emisión transferibles. La aplicación se realiza para el caso del Programa de Compensación de Emisiones (PCE) de la ciudad de Santiago de Chile en el año 1997. A diferencia de trabajos previos, el análisis de este artículo considera explícitamente, además de los costos de abatimiento de emisiones, los costos de fiscalización para inducir cumplimiento de la regulación. Específicamente, el propósito del trabajo es cuantificar los costos de cumplimiento de una regulación que reduce el nivel de emisiones agregadas en la ciudad de Santiago mediante un SPET, considerando tanto los costos de abatimiento como los costos de asegurar cumplimiento. Adicionalmente, se estima el impacto en términos de los costos de cumplimiento originados por una asignación inicial de permisos que difiere de aquella que minimiza los costos totales de cumplimiento.

La motivación central de este trabajo es responder dos preguntas fundamentales. Primero, en qué magnitud son subestimados los costos totales de la regulación cuando no se consideran los costos de fiscalización de una política ambiental basada en un SPET; segundo, en qué medida asignaciones iniciales de permisos no óptimas afectan la propiedad de costo-efectividad de un SPET, cuando existe poder de mercado y se consideran explícitamente los costos asociados a asegurar adecuados niveles de cumplimiento del programa.

En la sección 2 se presenta el marco conceptual que sirve de base para este trabajo. Se describe brevemente allí el modelo teórico desarrollado por Chávez y Stranlund (2003) sobre el cual se basan las simulaciones desarrolladas. En dicho trabajo los autores en primer lugar caracterizan una estrategia de fiscalización que es capaz de asegurar perfecto cumplimiento en el contexto de un SPET en presencia de poder de mercado. Considerando como dada dicha estrategia de fiscalización, en segundo lugar los autores caracterizan una asignación inicial óptima de permisos a la firma con poder de mercado cuando, además de considerar los cos- 
tos de abatimiento, se tienen en cuenta los costos de asegurar completo cumplimiento de la regulación. ${ }^{1}$

La sección 3 contiene la descripción de la metodología empleada para realizar las simulaciones que permiten la cuantificación de los costos de cumplimiento y de la magnitud de la pérdida de eficiencia derivada de una asignación inicial de permisos no óptima. En esta sección, proveemos inicialmente una breve descripción del PCE de la ciudad de Santiago así como de las fuentes que operaban bajo este programa en 1997. Posteriormente se presenta una discusión acerca de la especificación funcional del modelo teórico, con particular atención respecto de las funciones de costo marginal de abatimiento utilizadas en las simulaciones. Específicamente, se muestra aquí la forma en la cual, a partir de las funciones de costo marginal de abatimiento agregadas reportadas en la literatura, se obtienen las funciones de costo marginal de abatimiento para cada una de las fuentes consideradas. Finalmente se describe la solución analítica del modelo teórico así como la parametrización del mismo.

En la sección 4 reportamos los resultados obtenidos en nuestras simulaciones numéricas. Primero, se presentan y analizan los resultados obtenidos para las variables endógenas del modelo a partir de las simulaciones realizadas en el escenario base. Segundo, se presentan y comparan los resultados obtenidos con relación a los costos de cumplimiento en tres situaciones específicas: (1) asumiendo que existe competencia perfecta e ignorando los costos de inducir cumplimiento, (2) suponiendo la presencia de poder de mercado, pero ignorando nuevamente costos de inducir cumplimiento, y (3) considerando adicional a la presencia de poder de mercado, los costos de fiscalización para inducir cumplimiento de la regulación. Tercero, se reportan los resultados obtenidos con relación a la cuantificación del impacto en términos de pérdida de eficiencia ocasionada por una asignación inicial de permisos a la firma dominante diferente a la óptima. La sección concluye con la presentación de los resultados de un análisis de sensibilidad del valor de dicha pérdida de eficiencia frente a variaciones en los parámetros exógenos a las simulaciones.

Nuestros resultados muestran que los costos asociados a inducir cumplimiento de la regulación -costos de fiscalización y emisión de reportes, usualmente ignorados en la literatura- son importantes y su relevancia relativa dentro de los costos de cumplimiento depende en gran medida del objetivo ambiental deseado. Los resultados sugieren, además, que la presencia de poder de mercado produce un incremento modesto en los costos de cumplimiento, lo que indica un bajo impacto de la presencia de poder de mercado sobre los costos de cumplimiento para el caso del PCE. El modesto incremento en los costos de cumplimiento, obtenido en la simulación base a partir de asignaciones iniciales de permisos no

La determinación de la asignación inicial óptima es útil en el contexto de nuestro trabajo porque nos permite fijar un escenario base para responder una de las preguntas centrales, esta es, cuál es la magnitud de pérdida de eficiencia cuando se implementan asignaciones alternativas (no óptimas). Es interesante notar que debido a problemas de información que enfrenta el regulador, las asignaciones no óptimas de permisos constituyen el escenario más probable en el cual se desarrolla la regulación. 
óptimas, es robusto para diferentes niveles de los parámetros empleados en las simulaciones con la única excepción del parámetro referido a la meta ambiental deseada. Finalmente, en la sección 5 se presenta un análisis de los resultados obtenidos y las conclusiones que se desprenden de este trabajo.

\section{Poder de Mercado, Incentivos para Cumplimiento y Costos de la REGULACIÓN}

En esta sección presentamos el marco conceptual que sirve de base para el trabajo empírico. ${ }^{2}$ Se describe brevemente el modelo teórico desarrollado por Chávez y Stranlund (2003) sobre el cual se basan las simulaciones implementadas. ${ }^{3}$

\subsection{Incentivos para cumplimiento en presencia de poder de mercado}

El modelo teórico en el cual basamos las simulaciones numéricas considera la existencia de un conjunto de $\mathrm{n}$ firmas neutrales al riesgo participando en el mercado de permisos de emisión transferibles. La firma 1 (dominante) puede manipular el precio de los permisos, mientras que las restantes n-1 firmas son competitivas. La asignación inicial de permisos a la firma dominante se denota $\operatorname{como~}_{1}{ }^{0}$. El número de permisos que la firma dominante elige tener después de las transacciones se representa como $l_{1}$ y si el cumplimiento es garantizado, éste coincide con su nivel final de emisiones, $\mathrm{e}_{1}$. El número total de permisos disponibles en el mercado se denota como L y corresponde con la meta ambiental, es decir, el nivel agregado de emisiones que se espera alcanzar con la regulación. Las firmas tomadoras de precios demandan permisos de acuerdo con sus decisiones finales de emisión, $\mathrm{e}_{\mathrm{i}}$;

El modelo teórico que sirve de base para las simulaciones permite derivar estrategias de fiscalización apropiadas para inducir un nivel de cumplimiento dado, considerando poder de mercado sólo en el mercado de los permisos de emisión. Una preocupación alternativa está referida a la posibilidad de que haya firmas que manipulen el mercado de productos para ganar una posición estratégica en el mercado de productos [ver entre otros, Misiolek y Elder (1989), Innes, Kling, y Rubin (1991)]. Derivar una estrategia de fiscalización apropiada para dicho contexto y estudiar sus consecuencias desde el punto de vista de la característica de costo-efectividad de un SPET es, en nuestra opinión, una extensión interesante del trabajo teórico. Naturalmente, ello está fuera del alcance de este trabajo, considerando su naturaleza eminentemente empírica.

Otros autores que han examinado cómo la presencia de poder de mercado afecta aspectos de la fiscalización para inducir cumplimiento en un SPET son van Egteren y Weber (1996), y Malik (2002). Van Egteren y Weber realizan un análisis respecto del comportamiento de una firma en términos de su decisión de cumplimiento en presencia de poder de mercado, considerando la fiscalización como exógena. Malik (2002) analiza un mercado de permisos de emisión transferibles en el cual una firma tiene poder de mercado y una o más incurren en no-cumplimiento de la regulación. El autor muestra que el poder de mercado puede ser socialmente deseable, ya que puede mitigar los efectos negativos del no-cumplimiento de las firmas; de igual modo, el no-cumplimiento de las firmas puede ser socialmente deseable, ya que éste puede reducir los costos inducidos por la presencia de poder de mercado. 
este nivel final de emisiones coincide con la demanda final de permisos $1_{\mathrm{i}}$ si el cumplimiento es garantizado.

Una de las características del modelo es que asume la existencia de requerimiento de autorreportes de emisión a la autoridad reguladora de parte de las firmas reguladas. En este contexto, existen dos formas en que una firma podría encontrarse en violación: (i) transmitiendo datos de emisión erróneos (violación en reporte), y (ii) emitiendo una cantidad mayor al número de permisos que mantiene (violación en emisiones). Fiscalizar el programa para inducir cumplimiento, consiste en realizar auditorías para verificar las emisiones de las firmas, y en aplicar sanciones por violaciones en las emisiones y en los reportes, en caso de que éstas sean detectadas. Desde el punto de vista de la autoridad reguladora existen tres variables bajo su control: (i) monitoreo o auditorías para detectar la ocurrencia de violaciones, (ii) sanciones en caso de violación de reportes; y (iii) sanciones por emisiones en exceso de la tenencia de permisos.

En el contexto descrito, el modelo asume que cada firma elige el nivel de emisiones $\left(\mathrm{e}_{\mathrm{i}}\right)$, el nivel de reporte de emisiones $\left(\mathrm{r}_{\mathrm{i}}\right)$ y la demanda de permisos $\left(\mathrm{l}_{\mathrm{i}}\right)$ de manera de minimizar sus costos esperados de cumplimiento. Denotamos las siguientes variables:

$\mathrm{p}\left(\mathrm{L}-\mathrm{l}_{1}\right)$ : precio al cual se transan permisos (función del nivel total de permisos disponibles para las firmas competitivas).

$p\left(L-1_{1}\right)-p^{\prime}\left(L-1_{1}\right) \times\left(1_{1}-1_{1}^{0}\right)$ : precio efectivo de los permisos, esto es, el precio que enfrenta la firma con poder de mercado.

$1_{1}^{0}$ : $\quad$ asignación inicial de permisos a la firma con poder de mercado.

$\mathrm{p}^{\prime}\left(\mathrm{L}-\mathrm{l}_{1}\right)$ : pendiente de la demanda por permisos de las firmas competitivas.

$\pi_{\mathrm{i}}$ : $\quad$ probabilidad de que la firma i sea auditada.

f: $\quad$ sanción por unidad de violación de emisiones.

g: $\quad$ sanción por unidad de violación de reporte.

Inducir cumplimiento de la regulación requiere garantizar que las firmas entregarán un reporte verdadero de sus emisiones, y adicionalmente, asegurar que dichas firmas no emitirán una cantidad mayor al número de permisos que ellas poseen. Asumiendo, además, que en el caso de que una violación sea detectada las sanciones son aplicadas automáticamente, entonces Chávez y Stranlund (2003) muestran que en presencia de poder de mercado, la estrategia para inducir perfecto cumplimiento en un SPET está dada por las siguientes ecuaciones:

$$
\begin{aligned}
& \pi_{1}^{\text {min }}=\frac{\mathrm{p}\left(\mathrm{L}-\mathrm{l}_{1}\right)-\mathrm{p}^{\prime}\left(\mathrm{L}-\mathrm{l}_{1}\right) \times\left(\mathrm{l}_{1}-\mathrm{l}_{1}^{0}\right)}{\mathrm{g}+\mathrm{f}} \\
& \pi_{\mathrm{i}}^{\text {min }}=\frac{\mathrm{p}\left(\mathrm{L}-\mathrm{l}_{1}\right)}{\mathrm{g}+\mathrm{f}}, \mathrm{i}=2, \ldots, \mathrm{n} ; \\
& \mathrm{f} \geq \max \left[\mathrm{p}\left(\mathrm{L}-\mathrm{l}_{1}\right), \mathrm{p}\left(\mathrm{L}-\mathrm{l}_{1}\right)-\mathrm{p}^{\prime}\left(\mathrm{L}-\mathrm{l}_{1}\right) \times\left(\mathrm{l}_{1}-\mathrm{l}_{1}^{0}\right)\right]
\end{aligned}
$$


Las condiciones (1a) y (1b) establecen requerimientos mínimos de monitoreo que garantizan que cada firma emitirá reportes verdaderos. Dichos niveles de monitoreo son necesarios para garantizar que cada firma elegirá mantener un nivel de permisos igual al nivel de emisiones. La condición en (1c) garantiza que cada firma mantendrá un nivel de permisos suficiente para cubrir sus emisiones dado que éstas enfrentan incentivos apropiados para emitir reportes verdaderos. Para demostraciones formales y una discusión detallada de la intuición de estos resultados se sugiere consultar Chávez y Stranlund (2003).

\subsection{Poder de mercado, fiscalización y costos de cumplimiento}

En el modelo antes revisado, los costos de cumplimiento de la regulación (CT) corresponden a la suma de los costos agregados de abatimiento (CA) y los costos de monitoreo (M). Los costos agregados de abatimiento (CA) están compuestos por los costos de abatimiento de la firma dominante $\left(\mathrm{c}_{1}\right)$ y la suma de los costos de abatimiento de las firmas tomadoras de precio $\left(\mathrm{c}_{\mathrm{i}}\right)$, es decir, los costos agregados de abatimiento de las firmas competitivas, como se observa en la ecuación (2).

$$
\mathrm{CA}\left(\mathrm{l}_{1}^{0}\right)=\mathrm{c}_{1}\left(\mathrm{l}_{1}\left(\mathrm{l}_{1}^{0}\right)\right)+\sum_{\mathrm{i}=2}^{\mathrm{n}} \mathrm{c}_{\mathrm{i}}\left(\mathrm{l}_{\mathrm{i}}\left(\mathrm{l}_{1}^{0}\right)\right)
$$

$\mathrm{CT}=\mathrm{CA}\left(\mathrm{l}_{1}^{0}\right)+\mathrm{M}\left(\mathrm{l}_{1}^{0}\right) . \quad$ Cuando es posible asegurar completo cumplimiento los costos de fiscalización están determinados exclusivamente por los costos de monitoreo. Denotamos $w$ como el costo de una auditoría; entonces, los costos de monitoreo pueden escribirse como:

$$
\mathrm{M}\left(\mathrm{l}_{1}^{0}\right)=\mathrm{w}\left[\pi_{1}^{\min }\left(1_{1}^{0}\right)+(\mathrm{n}-1) \pi_{1}^{\min }\left(1_{1}^{0}\right)\right]
$$

En presencia de poder de mercado y considerando el objetivo de inducir perfecto cumplimiento de la regulación, los costos totales de cumplimiento de un SPET son entonces función de la asignación inicial de permisos; esto es,

La asignación inicial óptima de permisos a la firma con poder de mercado cuando se tienen en cuenta costos de fiscalización $\left(\mathrm{l}_{1}^{0 \text { opt cf }}\right)$, será aquella que minimice los costos totales de la regulación.

En este contexto, Chávez y Stranlund (2003) encuentran que la asignación inicial de permisos a la firma con poder de mercado que minimiza los costos totales del programa está determinada únicamente por la condición $\mathrm{CA}^{\prime}\left(\mathrm{l}_{1}^{0}\right)=\mathrm{M}^{\prime}\left(\mathrm{l}_{1}^{0}\right){ }^{4}$

Dicha condición es necesaria y suficiente, debido a que los costos de cumplimiento son estrictamente convexos en $1_{1}^{0}$, esto porque los costos agregados de abatimiento son estrictamente convexos en $1_{1}^{0}$ y los costos de monitoreo son convexos en $1_{1}^{0}$. Ver Chávez y Stranlund (2003). 
Específicamente, los autores muestran que tal condición implica que la asignación óptima de permisos a la firma dominante cuando se incluyen en el análisis los costos de fiscalización de la política $\left(1_{1}^{0 \text { opt cf }}\right)$, puede consistir en hacer participar a dicha firma como compradora o vendedora de permisos. La forma específica que adopte dicha participación depende directamente de si los costos de monitoreo son crecientes o decrecientes en la asignación inicial de permisos a la firma dominante. ${ }^{5}$ El poder de mercado puede ser utilizado para reducir los costos de fiscalización, debido a ello, la asignación inicial óptima bajo este esquema es aquella en la que se deja participar a la firma dominante, ya sea como compradora o vendedora en el mercado de permisos.

Los resultados previamente descritos sugieren que si se implementa una asignación inicial como sugiere Hahn (1984), de forma tal que la firma con poder de mercado no participe de las transacciones $\left(l_{1}^{0 \text { opt Hahn }}\right)$, sólo se está garantizando la minimización de los costos agregados de abatimiento. Por tanto, la asignación inicial óptima en un SPET en presencia de poder de mercado y cuando se tienen en cuenta los costos de fiscalización para inducir cumplimiento, no coincide en general con la asignación inicial óptima de permisos cuando no se tienen en cuenta dichos costos.

\section{Aplicación al PCE de Santiago de Chile: Aspectos Metodológicos}

Esta sección contiene el detalle metodológico de las etapas consideradas en la realización de las simulaciones. La sección se ordena de la siguiente forma: Inicialmente se presenta una breve descripción del PCE de la ciudad de Santiago y de las fuentes reguladas bajo este programa en el año 1997. Posteriormente y con base en el modelo teórico antes revisado, se presenta la especificación funcional requerida por el modelo teórico con la cual se implementan las simulaciones. ${ }^{6}$

De acuerdo con Chávez y Stranlund (2003), esto depende del grado de influencia de la firma dominante sobre el precio de los permisos, la pendiente de la función de costos marginales de abatimiento de la firma con poder de mercado, y el número de firmas que participan en el mercado.

6 El modelo teórico en el cual se basan las simulaciones supone cumplimiento perfecto de la regulación; no obstante, dados los costos de las inspecciones, el nivel de cumplimiento apropiado puede ser inferior a $100 \%$. La pregunta referida a cuál es el nivel apropiado de cumplimiento no está, en nuestro conocimiento, ni abordado ni resuelto en la literatura relacionada. El objetivo de cumplimiento perfecto considerado en el modelo teórico en que sustenta nuestro trabajo empírico, está basado en la experiencia práctica de algunos sistemas de permisos de emisión transferibles exitosos como el Programa de Lluvia Ácida - EPA $\mathrm{SO}_{2}$ - y el Programa Regional Clean Air Incentives Markets (RECLAIM) de Los Angeles, California, los cuales han logrado, respectivamente, perfecto cumplimiento, y una alta tasa de cumplimiento desde su implementación durante los años noventa. Esto sugiere que en la práctica es posible diseñar dichos sistemas con un objetivo de cumplimiento como el que supone en este trabajo. Anticipamos que estrategias de fiscalización consistentes con cumplimiento imperfecto resultarán en una reducción en el nivel de costos de cumplimiento de un SPET. Identi- 
Finalmente, se plantea la solución analítica al modelo teórico con las formas funcionales específicas y una descripción de los parámetros usados en las simulaciones.

3.1. El programa de compensación de emisiones (PCE) de la ciudad de Santiago

La descripción que a continuación se presenta está fundamentalmente basada en Montero et al. (2001) y Palacios y Chávez (2002). El Programa de Compensación de Emisiones (PCE) fue establecido por el Decreto Supremo No4 (DS 4) en marzo de 1992, para controlar las emisiones de material particulado total en suspensión (PTS) de fuentes estacionarias de origen industrial en la ciudad de Santiago de Chile. El DS 4 también instruyó al Servicio de Salud del Ambiente de la Región Metropolitana (SESMA) a través de su Oficina para el Control de Emisiones de las Fuentes Estacionarias (PROCEFF) (actual Subdepartamento de Calidad del Aire) para fiscalizar el Programa de Compensación. Las fuentes que estaban registradas cuando el DS 4 se promulgó (en adelante fuentes existentes) recibieron permisos de capacidad de emisión diaria a perpetuidad, de manera proporcional a una tasa de emisiones (concentración de emisiones) predeterminada y uniforme para todas las fuentes $\left(56 \mathrm{mg} / \mathrm{m}^{3}\right){ }^{7}$

Las fuentes bajo el PCE deben mantener suficientes permisos para cubrir el nivel máximo de emisiones proyectado, es decir, para cubrir la capacidad de emisión de la fuente. Esa capacidad de emisión es determinada con base en el tamaño de la fuente y el tipo de combustible que utiliza. El cumplimiento de la fuente se evalúa anualmente. Una fuente existente puede ser vendedora o compradora de permisos de capacidad de emisión dependiendo de si dicha capacidad está por debajo o sobre los permisos que le fueron asignados, respectivamente.

Las fuentes nuevas y las ampliaciones de fuentes existentes no reciben permisos y deben cubrir su capacidad de emisión adquiriendo éstos en el mercado.

ficamos dos efectos. Primero, el cumplimiento incompleto requiere de un menor requerimiento de fiscalización, reduciendo por tanto los costos relacionados. Segundo, bajo cumplimiento incompleto, las emisiones finales agregadas son mayores y por tanto el esfuerzo de abatimiento que realizan las fuentes es menor; esto implica una reducción en los costos de abatimiento. No obstante lo anterior, y quizá más importante, no es obvio el que, en este contexto, debiéramos esperar cambios significativos en las pérdidas de eficiencia estimadas, una de las preocupaciones centrales de nuestro trabajo empírico. Ello, debido a que el impacto sobre la eficiencia debe ser calculado controlando para un nivel dado de logro ambiental. Las estimaciones de impacto en eficiencia que nosotros presentamos están calculadas considerando cumplimiento perfecto, siendo ello consistente con las estrategias de fiscalización endógenas sugeridas por el modelo teórico. Agradecemos a dos árbitros anónimos de Cuadernos de Economía por motivar esta precisión.

7 Los permisos de capacidad de emisión diaria (EDI) fueron asignados según la fórmula: EDI $(\mathrm{kg} . /$ día $)=\mathrm{F}_{0}\left(\mathrm{~m}^{3} / \mathrm{hr}\right) * 56\left(\mathrm{mg} / \mathrm{m}^{3}\right) * 24(\mathrm{hr} / \mathrm{dí} a) * 10^{-6}(\mathrm{~kg} . / \mathrm{mg})$. Donde $\mathrm{F}_{0}$ es el máximo flujo de emisiones según lo declarado por la fuente al momento del registro en 1992. 
Cada permiso de capacidad máxima de emisión habilita para emitir un kilogramo diario a perpetuidad. El límite agregado de emisiones es la suma de los permisos asignados a las fuentes existentes.

Las fuentes existentes y nuevas, anualmente deben demostrar cumplimiento de la regulación mediante la entrega de un reporte al Subdepartamento de Calidad delAire. Dicho reporte debe incluir, entre otra información, una declaración del nivel de emisiones. Esta declaración de emisiones es contrastada con la cantidad de permisos que la fuente posee con el fin de determinar el estatus de cumplimiento de la fuente respecto de la regulación.

El SESMA se encarga de verificar la veracidad de las emisiones declaradas por la fuente a través de las auditorías y laboratorios privados de medición calificados previamente por éste. Si se detecta que una fuente emite una cantidad mayor a los permisos que posee, se ve sometida a la apertura de un sumario sanitario y un procedimiento que podría concluir en la aplicación de una sanción monetaria que fluctúa entre $\$ 2.850$ (US\$ 4,50 ) y $\$ 57.000 .000$ (US\$90,000) o la prohibición del funcionamiento de la fuente. ${ }^{8}$

Las simulaciones cuyos resultados son reportados en este artículo fueron realizadas utilizando la base de datos para el PCE correspondiente al año 1997, proporcionada por el SESMA. Del total de 870 fuentes presentadas en la base de datos original para el año 1997, fueron finalmente utilizadas en las simulaciones sólo aquellas fuentes que tenían información completa con relación a los siguientes campos de información: tipo de equipo, combustible utilizado, concentración de emisiones, permisos de capacidad máxima de emisión asignados, y emisiones diarias declaradas. El número de fuentes que tiene tal información completa asciende a 429 , de las cuales 414 son existentes y 15 son nuevas. Del total de 429 fuentes, 113 poseen equipo de abatimiento y 316 no poseen éste. En el Cuadro 1 se muestra una caracterización de las fuentes utilizadas en las simulaciones según el combustible y tipo de proceso que utilizan, en ella puede observarse que la mayoría de las fuentes utilizan petróleo como combustible (303 fuentes) y el generador de vapor es el proceso más utilizado dentro de las mismas (213 fuentes).

Cuando se examina la base de datos existente para dicho programa, se observa que en el año 1997 el porcentaje promedio de permisos de capacidad de emisión poseídos por una fuente es $0,23 \%$. Sin embargo, existe una fuente que posee el $11 \%$ de los permisos de capacidad de emisión. Esto confirma la concentración existente respecto de la propiedad de los permisos de capacidad de emisión, y es un indicio de la posible presencia de poder de mercado. ${ }^{9}$

8

Para una descripción detallada acerca del diseño de fiscalización del PCE, y resultados en términos de su cumplimiento, ver Palacios y Chávez (2002).

Respecto de este aspecto, es interesante notar la relativa alta concentración de permisos de capacidad de emisión observada en 1997. En efecto, de acuerdo a Montero et al. (2001) la agrupación de las fuentes según su propiedad utilizando el número del RUT mostraba que en 1997 el 50\% de los permisos totales era poseído por 21 firmas; mientras que 5 firmas poseían el $31 \%$ de los permisos. De acuerdo a estos autores, el mercado tiene un grado de concentración que puede explicar, en parte, la escasa oferta de derechos y su alto precio (Montero et al., 2001). 
CUADRO 1

CARACTERIZACION DE FUENTES UTILIZADAS EN LA SIMULACION

\begin{tabular}{lrlr}
\hline $\begin{array}{l}\text { Combustible } \\
\text { utilizado }\end{array}$ & $\begin{array}{c}\text { Número de } \\
\text { fuentes }{ }^{\text {a }}\end{array}$ & Tipo de proceso & $\begin{array}{c}\text { Número de } \\
\text { fuentes }{ }^{\text {b }}\end{array}$ \\
\hline Petróleo & 303 & Caldera agua caliente & 4 \\
Gas de cañería & 5 & Caldera de calefacción & 76 \\
Gas licuado & 14 & Caldera de calefacción y agua caliente & 7 \\
Kerosene & 12 & Caldera de fluido térmico & 3 \\
Aserrín & 7 & Caldera gas & 1 \\
Carbón bituminoso & 13 & Caldera generadora de vapor & 41 \\
Leña & 26 & Caldera industrial & 83 \\
Superlef & 33 & Caldera superior & 1 \\
LMFO 180 & 7 & Generador de vapor & 213 \\
Viruta & 1 & & \\
Skygard & 4 & & 429 \\
Chil & 2 & & \\
Gas natural & 2 & & \\
TOTAL & 429 & & \\
\hline
\end{tabular}

Fuente: Elaboración propia a partir de base de datos proporcionada por el SESMA para fuentes en el PCE (1997).

Notas:

a Número de fuentes que utilizan cada tipo de combustible.

${ }^{\mathrm{b}}$ Número de fuentes que tienen cada tipo de proceso.

\subsection{Funciones de costo marginal de abatimiento}

La cuantificación de los costos de cumplimiento y de la pérdida de eficiencia ocasionada por una asignación inicial de permisos diferente a la óptima, cuando se considera poder de mercado, y la necesidad de inducir cumplimiento de la regulación, es calculada usando las funciones de costos marginales agregadas anuales para fuentes existentes y fuentes nuevas del PCE en Santiago en el año de 1997, reportadas por Montero et al. (2002). ${ }^{10}$ Estas funciones fueron construidas por los autores basados en información de la literatura y en entrevistas a los operadores de las industrias y vendedores de equipos de control (ver Montero et al., 2001). El uso de tales funciones presenta la limitación de que son agregadas y no permiten captar la heterogeneidad en los costos marginales de abatimiento existente entre las fuentes. Debido a ello, para el desarrollo del presente trabajo se utilizan las funciones mencionadas como referencia, intentando captar la heterogeneidad entre las fuentes según se explica a continuación. 
La obtención de las funciones de costo marginal de abatimiento para cada una de las fuentes a partir de las funciones de costos marginales de abatimiento agregadas, requirió primero obtener una función base de costos marginales de abatimiento para las fuentes existentes y nuevas $\left(\mathrm{CMb}_{\mathrm{E}}\right.$ y $\mathrm{CMb}_{\mathrm{N}}$, respectivamente). Con posterioridad, se incorporó a dicha función base un factor de corrección que intenta captar la heterogeneidad desde el punto de vista de costos de abatimiento entre las fuentes. Este factor de corrección depende de las características del proceso tipo al cual pertenece la fuente. ${ }^{11}$

La determinación del proceso tipo al cual pertenece cada fuente se realizó según se describe a continuación. Inicialmente, y con base en información disponible en la base de datos del PCE para el año definido, se clasificaron las fuentes como existentes o nuevas. Posteriormente, en un procedimiento análogo al utilizado por Villena y Villena (1998), y dado que los costos marginales de abatimiento están muy relacionados con el tipo de tecnología que se utiliza, las fuentes existentes y nuevas se subclasifican en 74 "procesos tipo" para las fuentes existentes y 6 "procesos tipo" para las fuentes nuevas. El procedimiento considera el proceso y el tipo de combustible utilizado. Así por ejemplo, un "proceso tipo" son todas aquellas fuentes existentes que utilizan generador de vapor y gas de cañería. De esta forma se obtienen entonces, partiendo de un total de 429 fuentes, 80 "procesos tipo". En el Anexo 1 se presenta la descripción de los "procesos tipo" para las fuentes consideradas.

Específicamente, las funciones base de costo marginal de abatimiento para fuentes existentes y nuevas, respectivamente, son:

$$
\mathrm{CMb}_{\mathrm{E}}=360+1.05 \times \mathrm{n}_{\mathrm{E}} \times\left(\mathrm{e}_{\mathrm{iE}}^{0}-\mathrm{e}_{\mathrm{iE}}\right)
$$

$$
\mathrm{CMb}_{\mathrm{N}}=23.73 \times \mathrm{n}_{\mathrm{N}} \times\left(\mathrm{e}_{\mathrm{iN}}^{\mathrm{o}}-\mathrm{e}_{\mathrm{iN}}\right)
$$

Donde $\mathrm{CMb}_{\mathrm{E}}$ y $\mathrm{CMb}_{\mathrm{N}}$ representan el costo marginal de abatimiento base para fuentes existentes y nuevas, respectivamente; $\mathrm{n}_{\mathrm{E}} \mathrm{y} \mathrm{n}_{\mathrm{N}}$ es el número de fuentes existentes y nuevas, respectivamente; $\mathrm{e}_{\mathrm{iN}}^{0}$ y $\mathrm{e}_{\mathrm{iN}}^{0}$ representan las emisiones ini-

11 El procedimiento es análogo al utilizado por Westskog (1996) quien para calcular la pérdida de eficiencia por poder de mercado en un sistema de permisos de emisión transferibles de $\mathrm{CO}_{2}$ entre países, utiliza una función de costos marginales de abatimiento como referencia; esta función presenta la limitación de no tener en cuenta que los costos difieren entre países cuando ellos quieren reducir el mismo porcentaje de emisiones, diferencias que pueden deberse a los distintos sistemas de energía o uso de tecnología. Para tener en cuenta tales diferencias, inicialmente Westskog clasifica los países en tres categorías diferentes dependiendo de la intensidad de carbón que utilizan (países que tienen una intensidad menor, igual o mayor a $0.26 \mathrm{~kg} / \$$ ) y a la función de costos marginales de abatimiento de referencia, le incorpora un factor de corrección que es diferente para cada país ya que depende de la intensidad de carbón que cada uno utiliza. Este factor de corrección aumenta los costos marginales de abatimiento cuando el país tiene una intensidad de carbón inferior al parámetro base, y los disminuye cuando tienen una intensidad superior a tal parámetro. 
ciales de las fuentes existentes y nuevas (emisiones sin regulación); y $\mathrm{e}_{\mathrm{iE}} \mathrm{ye}_{\mathrm{iN}}$ son, respectivamente, las emisiones finales seleccionadas por cada una de las fuentes existentes y nuevas. Adicionalmente, tanto para el caso de fuentes existentes como nuevas, las emisiones iniciales corresponden a las emisiones reportadas por cada fuente en el año $1997 .^{12}$

El factor de corrección aplicado a las funciones base de costo marginal de abatimiento fue construido utilizando información de concentración de las emisiones, expresada en $\mathrm{mg} / \mathrm{m}^{3}$, de cada uno de los procesos tipo identificados. El valor de este factor de corrección es diferente para cada una de las fuentes ya que depende directamente de la concentración de cada una de ellas. El factor de corrección para cada fuente $\mathrm{i}\left(\mathrm{FC}_{\mathrm{i}}\right)$ se define como

$$
\mathrm{FC}_{\mathrm{i}}=\frac{\mathrm{C}_{\mathrm{i}}}{\mathrm{C}_{\mathrm{j}}}
$$

Donde i indexa la fuente; $\mathrm{j}$ indexa el "proceso tipo" al cual pertenece la fuente; $\mathrm{C}_{\mathrm{i}}$ representa la concentración de la fuente $\mathrm{i} ; \mathrm{C}_{\mathrm{j}}$ es el promedio ponderado de las concentraciones de las fuentes pertenecientes al "proceso tipo" $\mathrm{j}$ al cual pertenece la fuente i. ${ }^{13}$ Las funciones base de costo marginal de abatimiento se corrigen entonces con este factor y se obtiene la función de costo marginal de abatimiento para cada una de las fuentes existentes y nuevas, respectivamente. Estas son:

$$
\begin{aligned}
& \mathrm{CMg}_{\mathrm{iE}}=360+\frac{1.05 \times \mathrm{n}_{\mathrm{E}} \times \mathrm{e}_{\mathrm{iE}}^{0}}{\mathrm{FC}_{\mathrm{i}}}-\frac{1.05 \times \mathrm{n}_{\mathrm{E}} \times \mathrm{e}_{\mathrm{i}}}{\mathrm{FC}_{\mathrm{i}}} \\
& \mathrm{CMg}_{\mathrm{iN}}=\frac{23.73 \times \mathrm{n}_{\mathrm{N}} \times \mathrm{e}_{\mathrm{iN}}^{0}}{\mathrm{FC}_{\mathrm{i}}}-\frac{23.73 \times \mathrm{n}_{\mathrm{N}} \times \mathrm{e}_{\mathrm{i}}}{\mathrm{FC}_{\mathrm{i}}}
\end{aligned}
$$

Las ecuaciones (7) y (8) sugieren una especificación lineal de la forma $\mathrm{CMg}_{\mathrm{iE}}=\mathrm{b}_{\mathrm{iE}}+\mathrm{a}_{\mathrm{iE}} \times \mathrm{e}_{\mathrm{i}} ; \mathrm{y} \mathrm{CMg}_{\mathrm{iN}}=\mathrm{b}_{\mathrm{iN}}+\mathrm{a}_{\mathrm{iN}} \times \mathrm{e}_{\mathrm{i}}$, para fuentes existentes y nuevas, respectivamente. Adicionalmente, en dicha especificación el factor de corrección incorporado tiene el mismo efecto que el que usa Westskog (1996) en su trabajo; esto es, aumenta los costos marginales de abatimiento de aquellos procesos tipos cuyo promedio de concentración es menor que el promedio de concentración de todas las fuentes $(\mathrm{FC}<1)$; y disminuye aquellos para los cuales el promedio de concentración es mayor que el de todas las fuentes $(\mathrm{FC}>1)$. La intuición detrás del procedimiento antes descrito es la siguiente: si una fuente invierte un monto determinado de recursos para el control de la contaminación de una unidad de emisiones, por ejemplo una tonelada, los costos de abatimiento de dicha unidad abatimiento se presenta en el Anexo 2. 
serán menores a mayor concentración de contaminante, lo anterior porque el nivel de flujo asociado a dicha unidad de emisiones es menor. ${ }^{14}$

\subsection{Solución analítica y selección de parámetros}

Se desarrolló una solución analítica para el modelo general de fiscalización en presencia de poder de mercado de Chávez y Stranlund (2003) revisado en la sección 2. En dicha solución analítica, se derivaron expresiones que: i) definen la dotación inicial óptima de permisos a la fuente con poder de mercado; ii) describen la selección de emisiones por parte de la fuente con poder de mercado y de las fuentes competitivas; iii) caracterizan las decisiones de cumplimiento por parte de las fuentes, es decir, aquellas que inducen a cada fuente a emitir un reporte verdadero de emisiones y emitir una cantidad de contaminante igual al número de permisos que poseen; iv) determinan las probabilidades mínimas de auditar e imponer sanciones necesarias para garantizar completo cumplimiento; y finalmente v) permiten calcular los costos marginales y totales de abatimiento y de fiscalización, y consecuentemente, los costos de cumplimiento.

Luego de obtenida la solución analítica, se procedió a definir los parámetros a ser utilizados en las simulaciones. Describimos a continuación brevemente la elección de dichos parámetros. Los valores de éstos aquí presentados constituyen el escenario base para la realización de las simulaciones.

La determinación de la fuente dominante se realiza de manera análoga a Westskog (1996); es decir, se identifica como dominante a la fuente que tiene el mayor porcentaje de las emisiones totales. Específicamente, en el caso del PCE, y considerando el año 1997, dicha fuente posee un porcentaje de las emisiones totales que asciende a un $11.56 \%$. En las simulaciones realizadas se considera ésta como la fuente dominante. ${ }^{15}$

Los valores de los parámetros de la función de costos marginales de abatimiento para la fuente con poder de mercado, esto es, $\mathrm{a}_{1} \mathrm{y}_{1}$ en la especificación lineal que sugiere la ecuación (7), son calculados utilizando dicha especificación funcional y la información provista en la base de datos del PCE. De igual forma, los parámetros de la función de costo marginal de abatimiento para fuentes competitivas, esto es, $a_{i}$ y $b_{i}$, se calculan utilizando las especificaciones funcionales sugeridas en (7) y (8) y la información contenida en la base de datos. Estos parámetros determinan la función de demanda por permisos por parte de las fuentes competi- 
tivas. Esta función, que es obtenida a partir de la suma de las demandas individuales lineales, tiene una especificación también lineal de la forma: $\mathrm{d}+\mathrm{k} \times \mathrm{p} .{ }^{16}$

Las emisiones agregadas finales que se tienen como objetivo de política (L) se obtienen suponiendo una meta de reducción de emisiones de 50\% y considerando como emisiones agregadas iniciales aquellas declaradas en el año de 1997 $(2,883 \mathrm{~kg} / \mathrm{día})$. Estas emisiones agregadas finales corresponden con el número de permisos de emisión presentes en el mercado. Para el escenario base, el número total de permisos existentes (L) es de 1,442 kg/día. Con relación al valor de las sanciones por violaciones, la sanción marginal por violación de emisiones (f) se supone en un nivel equivalente al máximo entre el precio y el precio efectivo del permiso. Se hace esta consideración teniendo en cuenta la estructura de sanciones del PCE de la ciudad de Santiago descrita en la sección 3.1, y considerando que la imposición de sanciones en este programa no ha sido estricta. Este es precisamente uno de los parámetros para el cual se realiza un análisis de sensibilidad. Similar al diseño del PCE, no se considera ninguna sanción para el caso de violación en los reportes.

El costo unitario de las auditorías (w) se obtuvo considerando el costo de un muestreo isocinético. Este asciende a aproximadamente US\$ 870 (dólares del año 1998). Este valor se incrementó en un $40 \%$ para tener en cuenta los costos indirectos relacionados con la auditoría. El costo final de la auditoría es entonces alrededor de US\$ $1,217 .{ }^{17}$

Considerando las expresiones derivadas de la solución analítica del modelo y los parámetros correspondientes a las funciones de costos marginales de abatimiento de las fuentes $\left(a_{i}\right.$ y $\left.b_{i} ; i=1, . . n\right)$, las emisiones agregadas que se tienen como meta (L), el valor de las sanciones marginales por violaciones de emisiones (f), el costo de una auditoría (w), el número de fuentes que participan en el mercado (n) y los parámetros de la función de demanda por permisos por parte de las firmas competitivas ( $\mathrm{d}$ y k), se calculó la asignación inicial óptima a la fuente con poder de mercado $\left(1_{1}^{0 \text { optef }}\right)$, el precio de mercado y el precio efectivo de los permisos (precio que enfrenta la fuente con poder de mercado), la elección del nivel de emisión de cada fuente, los requerimientos mínimos de monitoreo a la fuente con poder de mercado $\left(\pi_{1}^{\text {min }}\right)$ y a las fuentes competitivas $\left(\pi_{i}^{\text {min }}\right)$, los costos agregados de abatimiento (CA), los costos de fiscalización (M), y los costos de cumplimiento. Los costos agregados de abatimiento (CA) se calculan como la suma de los costos totales de abatimiento para cada una de las fuentes. Estos costos totales de abatimiento para cada una de las fuentes se calculan a partir de la especificación funcional de los costos marginales de abatimiento considerando el nivel inicial y el nivel demanda por permisos por parte de las fuentes competitivas. 
final de emisiones seleccionado por cada una de las fuentes. Los costos de cumplimiento del programa corresponden a la suma de los costos de fiscalización y los costos de abatimiento.

\section{Resultados de las Simulaciones}

En esta sección se reportan los principales resultados obtenidos a partir de las simulaciones. Primero, se muestran los resultados obtenidos en el escenario base para las variables endógenas consideradas. Segundo, se comparan los resultados para diferentes tipos de costos de cumplimiento de la regulación. Tercero, se presentan también los resultados obtenidos con relación a la cuantificación del impacto en términos de pérdida de eficiencia ocasionada por una asignación inicial de permisos a la firma dominante diferente a la óptima. Las simulaciones realizadas son estáticas; es decir, sólo se consideran los costos del programa para el año 1997. Todos los valores monetarios están expresados en dólares del año 1998.

\subsection{Resultados de las simulaciones en el escenario base}

Se realizaron las simulaciones para el caso en el cual sólo se considera poder de mercado y el caso en el cual se consideran tanto la presencia de poder de mercado como los costos de fiscalización para inducir cumplimiento. Las simulaciones fueron realizadas para diferentes asignaciones iniciales. Se evaluaron asignaciones iniciales mayores y menores a la óptima. Dentro de estas asignaciones se estudiaron dos casos extremos de asignación: cuando no se le asigna ningún permiso a la fuente dominante $\left(1_{1}{ }^{0}=0\right)$ y cuando se le asignan todos los permisos a la fuente dominante $\left(1_{1}{ }^{0}=\mathrm{L}\right)$. Una síntesis de los resultados obtenidos es provista en el Cuadro 2.

\subsubsection{Asignación inicial óptima y demanda de permisos de la fuente dominante}

La dotación inicial óptima de permisos cuando sólo se considera poder de mercado consiste en asignar a la fuente dominante 325 permisos, un número de

permisos igual al que la fuente elegirá tener después de las transacciones. En el caso en el que, además de poder de mercado se consideran los costos de fiscalización, la asignación óptima inicial a la fuente con poder de mercado asciende a 243 permisos. Dado que la fuente elige tener 325 permisos después de las transacciones, la asignación inicial óptima cuando se consideran costos de fiscalización, debiera inducir a la fuente dominante a actuar como compradora de permisos en el mercado.

En el caso en el que se considera sólo poder de mercado así como cuando se consideran ambos, poder de mercado y costos de fiscalización, los resultados sugieren que la fuente con potencial poder de mercado no modifica en forma significativa el número de permisos que desea tener después de las transacciones ante variaciones en la asignación inicial de permisos. Cuando el número de permi- 


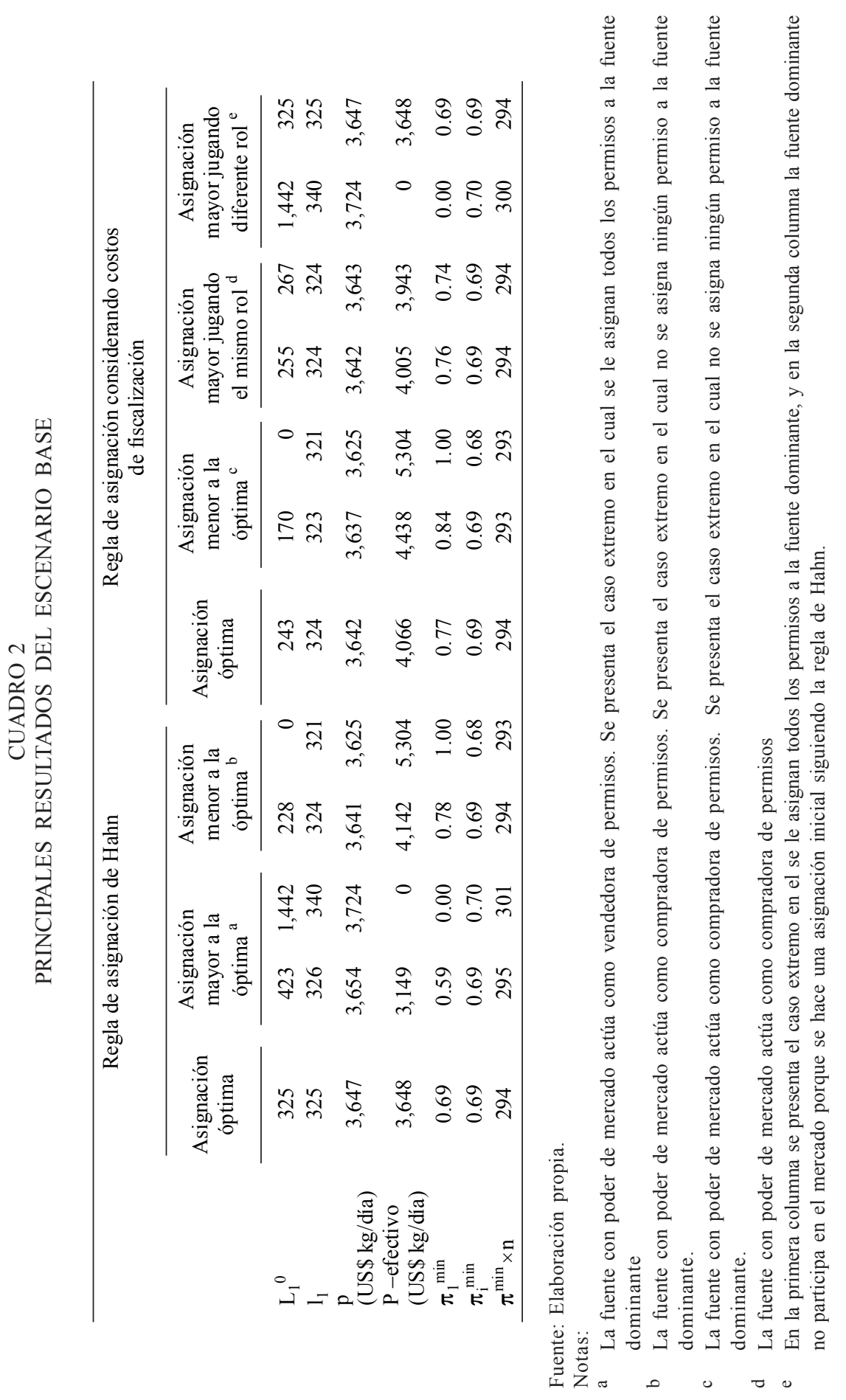


sos que recibe inicialmente la fuente dominante es mayor que el número que recibiría en la asignación óptima, dicha fuente elige demandar un mayor número de permisos. Lo contrario sucede cuando la asignación inicial de permisos es menor que la óptima. En dicho caso el nivel de emisiones finales de la fuente será menor que el que tendría con una asignación inicial óptima (ver Cuadro 2, columnas de asignación inicial diferente a la óptima).

El número mínimo de permisos que la fuente dominante elige demandar (321) se presenta cuando inicialmente no se le asigna ningún permiso; el número máximo de permisos que elige poseer (340), se presenta cuando inicialmente se le asignan todos los permisos.

\subsubsection{Precio de equilibrio y precio efectivo de los permisos}

En el caso en el cual se considera sólo poder de mercado, dada una asignación inicial óptima (325 permisos), el precio de los permisos es aproximadamente US\$ 3,647 kg/día. ${ }^{18}$ Cuando la asignación inicial es mayor que la óptima y la fuente con poder de mercado por consiguiente actúa como vendedora, el precio de equilibrio de los permisos aumenta. Lo anterior se debe a que con una asignación inicial a la fuente dominante mayor que la óptima, ésta elige mantener un número mayor de permisos después de las transacciones, haciendo que disminuya la oferta de permisos a fuentes competitivas y aumente el precio de equilibrio. De manera análoga, con una asignación inicial a la fuente dominante menor que la óptima, el precio de equilibrio de los permisos disminuye.

En el caso en el cual, además de tener en cuenta la presencia de poder de mercado se consideran los costos de fiscalización, si se hace una asignación inicial óptima, el precio de los permisos es aproximadamente US\$3,642 kg/día. Similar al caso en que sólo se considera poder de mercado, alteraciones en la asignación inicial óptima producen cambios, en el mismo sentido, aunque en diferentes magnitudes, en el precio de equilibrio de los permisos.

El precio efectivo de los permisos es aquel que enfrenta la fuente dominante. Cuando sólo se considera poder de mercado, y la asignación inicial es óptima, el precio efectivo de los permisos es de US\$3,648 kg/día. Contrario a lo que sucede con el precio de equilibrio, ante una asignación inicial menor (mayor) que la óptima, el precio efectivo de los permisos aumenta (disminuye).

En el caso en el cual además de tener en cuenta el poder de mercado se tienen en cuenta los costos de fiscalización, el precio efectivo para la asignación inicial óptima en la cual la fuente dominante actúa como compradora, es de US\$4,066 kg/día. Igual que en el caso anterior, para una asignación inicial menor (mayor) que la óptima, el precio efectivo de los permisos aumenta (disminuye). 
Se observa en los resultados que mientras mayor sea la diferencia entre la asignación inicial óptima y la asignación inicial que se hace de los permisos, mayor será la diferencia entre el precio de equilibrio y el precio efectivo de los mismos.

\subsubsection{Esfuerzo de fiscalización}

Dado que las sanciones marginales por violación se suponen constantes y uniformemente aplicadas, el requerimiento mínimo de monitoreo a las fuentes dominante y competitivas está directamente relacionado con el precio de equilibrio y el precio efectivo de los permisos, respectivamente. En efecto, ante un aumento en el precio de equilibrio o el precio efectivo, se incrementa el requerimiento mínimo de monitoreo que induce perfecto cumplimiento de parte de las fuentes competitivas o de la fuente dominante. En los dos casos estudiados, cuando a la fuente dominante se le asigna una cantidad inicial de permisos menor que la óptima, tal fuente debe ser auditada con una probabilidad mayor que la requerida en el caso de una asignación inicial óptima. Dicho resultado es esperado ya que si se le asignan menos permisos a la fuente dominante, el precio efectivo de los permisos aumenta y el beneficio marginal por una violación de emisiones será mayor, y consecuentemente, se requiere un mayor esfuerzo de monitoreo a la fuente con poder de mercado para garantizar cumplimiento. En este caso, las firmas competitivas tienen más permisos disponibles en el mercado, lo que hace que el precio de equilibrio de los permisos disminuya y con él su beneficio marginal de violar, por tanto, el requerimiento mínimo de monitoreo para inducir cumplimiento disminuye con respecto al caso de asignación inicial óptima.

En el caso en el cual se simula una asignación inicial de permisos mayor que la óptima, las fuentes competitivas tienen una menor oferta de permisos en el mercado; esto hace que el precio de los mismos aumente y con ello también lo haga el beneficio marginal de una violación de emisiones. Ello requiere que sea necesario un mayor esfuerzo de monitoreo a las fuentes competitivas para garantizar cumplimiento y un menor esfuerzo de monitoreo a la fuente dominante. El requerimiento mínimo de monitoreo a la fuente dominante alcanza su nivel más alto cuando inicialmente no se le asigna ningún permiso a ésta. El requerimiento mínimo de monitoreo sobre dicha fuente se observa cuando en la asignación inicial se le otorgan todos los permisos existentes. De manera análoga, el requerimiento mínimo de monitoreo a las fuentes competitivas se presenta cuando inicialmente no se otorga ningún permiso a la fuente dominante, y el más alto, cuando en la asignación inicial se dan todos los permisos a la fuente con poder de mercado.

\subsection{Costos de cumplimiento}

Se presentan aquí los resultados obtenidos para diferentes conceptos de costos de cumplimiento de la regulación. Se estudia a través de simulaciones cómo varían los costos bajo diferentes niveles objetivo de abatimiento agregado. Esta comparación tiene como propósito observar el impacto de ignorar la fiscalización 
para inducir cumplimiento y presencia de poder de mercado sobre los costos de la regulación y cómo varían dichos impactos con los niveles de abatimiento objetivos.

Los casos considerados son:

1. Competencia perfecta. En este caso, los costos totales de la regulación $\left(\mathrm{CT}_{\mathrm{cp}}\right)$ son los costos agregados de abatimiento de las fuentes participantes en el mercado, esto es, $\mathrm{CT}_{\mathrm{cp}}=\mathrm{CA}$.

2. Poder de mercado. Cuando sólo se considera la presencia de poder de mercado, los costos totales del programa $\left(\mathrm{CT}_{\mathrm{pm}}\right)$, al igual que en el caso anterior, se consideran como los costos agregados de abatimiento de las fuentes, es decir, la sumatoria de los costos de abatimiento de todas las fuentes participantes, de acuerdo a la ecuación (2). Estos costos de abatimiento en este caso, son función de la asignación inicial de permisos a la fuente con poder de mercado.

3. Poder de mercado y costos de fiscalización. En este caso, los costos totales del programa $\left(\mathrm{CT}_{\mathrm{cf}}\right)$ están determinados por la suma de los costos agregados de abatimiento y los costos de fiscalización (que están representados por los costos de monitoreo). Los costos de abatimiento y los costos de monitoreo son función de la asignación inicial de permisos a la firma dominante. Se incluyen además los costos en los que incurren las firmas para emitir sus reportes (CER), que están representados por el costo que tiene realizar una medición por parte de un laboratorio calificado para ello. Estos costos de emitir reportes son independientes del nivel de abatimiento y de la asignación inicial de permisos.

$$
\mathrm{CT}_{\mathrm{cf}}=\mathrm{CA}\left(1_{1}^{0}\right)+\mathrm{M}\left(1_{1}^{0}\right)+\mathrm{CER}
$$

En el Cuadro 3 se muestran los costos de cumplimiento que se obtienen, en los tres escenarios, a partir de las simulaciones considerando una asignación inicial de permisos a la firma dominante diferente a la óptima. En general, se considera poder de mercado los costos de la regulación cuando son levemente mayores que para el caso de competencia perfecta. Adicionalmente, en la situación en que, además de considerar poder de mercado, se consideran costos de fiscalización y de emitir reportes, los costos de la regulación son mayores que en los dos casos anteriores. En el Cuadro 3 se presentan los costos para diferentes niveles de abatimiento, desde 10\% como meta de descontaminación ambiental hasta 90\%. Cuando se considera, por ejemplo, un nivel de abatimiento del $50 \%$, los costos considerando competencia perfecta son de US\$ 2.29 millones. Dado el referido objetivo ambiental, dicho resultado es muy similar a la situación cuando se considera poder de mercado. En la situación en la cual se consideran, además de la presencia de poder de mercado, los costos de fiscalización y de emisión de reportes, los costos de cumplimiento de la meta de $50 \%$ de reducción de emisiones ascienden a US\$ 3.33 millones; nivel equivalente a 1.5 veces los costos de cumplimiento ignorando los costos de fiscalización. 


\begin{tabular}{|c|c|c|c|c|}
\hline Abatimiento ${ }^{a}$ & $\begin{array}{c}\mathrm{CT}_{\mathrm{cp}}{ }^{\mathrm{b}} \\
\text { (miles de dólares) }\end{array}$ & $\begin{array}{c}\mathrm{CT}_{\mathrm{pm}}{ }^{\mathrm{c}} \\
\text { (miles de dólares) }\end{array}$ & $\begin{array}{c}\mathrm{CT}_{\mathrm{cf}}^{\mathrm{d}} \\
\text { (miles de dólares) }\end{array}$ & $\begin{array}{l}\text { Indice de aumento } \\
\text { en los costos }\end{array}$ \\
\hline 10 & 149.1 & 149.2 & 1,107 & 7.4 \\
\hline 20 & 413.0 & 413.2 & 1,391 & 3.4 \\
\hline 30 & 820.6 & 820.9 & 1,820 & 2.2 \\
\hline 40 & $1,417.6$ & $1,418.1$ & 2,439 & 1.7 \\
\hline 50 & $2,286.3$ & $2,287.5$ & 3,331 & 1.5 \\
\hline 60 & $3,623.4$ & $3,628.2$ & 4,668 & 1.3 \\
\hline 70 & $5,952.8$ & $6,011.4$ & 7,018 & 1.2 \\
\hline 80 & $11,270.8$ & $11,402.4$ & 12,397 & 1.1 \\
\hline 90 & $21,896.1$ & $22,112.9$ & 23,100 & 1.0 \\
\hline
\end{tabular}

Fuente: Elaboración propia.

Notas:

a El nivel de abatimiento está expresado en porcentaje de reducción de emisiones

b Costos totales de lograr cumplimiento en competencia perfecta. Expresados en miles de dólares

c Costos totales de lograr cumplimiento en presencia de poder de mercado. Expresados en miles de dólares

d Costos totales de lograr cumplimiento en presencia de poder de mercado e incluyendo costos de fiscalización. Expresados en miles de dólares.

e Este índice muestra el número de veces en el que aumentaron los costos de cumplimiento cuando se tienen en cuenta los costos de fiscalización y de emitir reportes. Se calcula como $\mathrm{CT}_{\mathrm{cf}} / \mathrm{CT}_{\mathrm{cp}}$

Los resultados reportados en el Cuadro 3 sugieren que la magnitud en la cual se subestiman los costos de cumplimiento cuando no se consideran los costos de fiscalización es menor a medida que se incrementan las exigencias del programa. Específicamente, cuando se tienen metas ambientales muy estrictas, los costos de fiscalización y emisión de reportes representan un porcentaje reducido dentro de los costos totales de cumplimiento.

\subsection{Pérdidas de eficiencia}

La cuantificación de la magnitud de las pérdidas de eficiencia requiere calcular los costos totales en los que se incurre cuando, además de considerar la presencia de poder de mercado, se consideran los costos de fiscalización para inducir cumplimiento. Con los valores de los parámetros de entrada para el escenario base, se realizaron las simulaciones para obtener los costos totales de cumplimiento del programa para diferentes asignaciones iniciales, como se describió anteriormente, y a través de su comparación con los costos de cumplimiento para una asignación inicial óptima, conocer la magnitud de la pérdida de eficiencia. En el Cuadro 4 se presentan los resultados obtenidos cuando sólo se considera la presencia de poder de mercado, y la regla de asignación inicial utilizada está basada en Hahn (1984). 
CUADRO 4

PERDIDAS DE EFICIENCIA CUANDO SOLO SE CONSIDERA PODER DE MERCADO. ESCENARIO BASE

\begin{tabular}{|c|c|c|c|c|c|}
\hline & \multirow{2}{*}{ 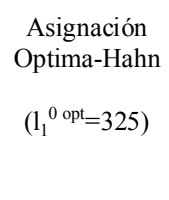 } & \multicolumn{4}{|c|}{ Asignación diferente a la óptima } \\
\hline & & $\begin{array}{c}\text { Mayor a la } \\
\text { óptima en } \\
30 \% \\
\left(1_{1}^{0}=423\right)\end{array}$ & $\begin{array}{c}\text { Mayor a la } \\
\text { óptima -caso } \\
\text { extremo- } \\
\left(1_{1}^{0}=1442\right)\end{array}$ & $\begin{array}{c}\text { Menor a la } \\
\text { óptima en } \\
30 \% \\
\left(1_{1}^{0}=228\right)\end{array}$ & $\begin{array}{c}\text { Menor a la } \\
\text { óptima -caso } \\
\text { extremo- } \\
\left(\mathrm{l}_{1}^{0}=0\right)\end{array}$ \\
\hline Costos de Cumplimiento a & 2,286 & 2,287 & 2,318 & 2,287 & 2,290 \\
\hline Pérdida de eficiencia (\%) ${ }^{b}$ & & 0.014 & 1.848 & 0.014 & 0.154 \\
\hline
\end{tabular}

Fuente: Elaboración propia.

Notas:

a Costos considerando sólo poder de mercado (en miles de dólares).

b Expresada en porcentaje.

Cuando la distribución inicial de permisos se realiza siguiendo una regla de asignación diferente a la óptima determinada por Hahn, y sólo se están considerando los costos de abatimiento, se produce una pérdida de eficiencia modesta. La magnitud de la pérdida de eficiencia es máxima $(1,85 \%)$ cuando en la asignación inicial se asignan todos los permisos a la fuente con poder de mercado.

En el Cuadro 5 se presentan los resultados cuando se consideran costos de abatimiento y costos de fiscalización, y la regla de asignación inicial utilizada es la adecuada en este caso. Los resultados reflejan que la pérdida de eficiencia depende de la manera en la cual se haga la asignación inicial de los permisos: cuando la asignación inicial es óptima se tiene la condición de costo-efectividad. Cuando se asignan inicialmente los permisos de manera diferente a esa regla de asignación, se obtiene una pérdida de eficiencia, es decir, los costos totales del programa se incrementan. Si se consideran costos de fiscalización y se hace una asignación inicial de permisos siguiendo la regla de Hahn, es decir, cuando la firma con poder de mercado no participa en las transacciones, se incurre también en una pérdida de eficiencia.

Al igual que cuando sólo se considera poder de mercado, la magnitud de la pérdida de eficiencia es extremadamente baja, y se incrementa a medida que aumenta el porcentaje de variación de la asignación inicial con respecto a la asignación inicial óptima. La máxima pérdida de eficiencia se obtiene para el caso extremo en el cual se asignan todos los permisos a la fuente con poder de mercado; dicha magnitud en este caso es $1,45 \%$. 


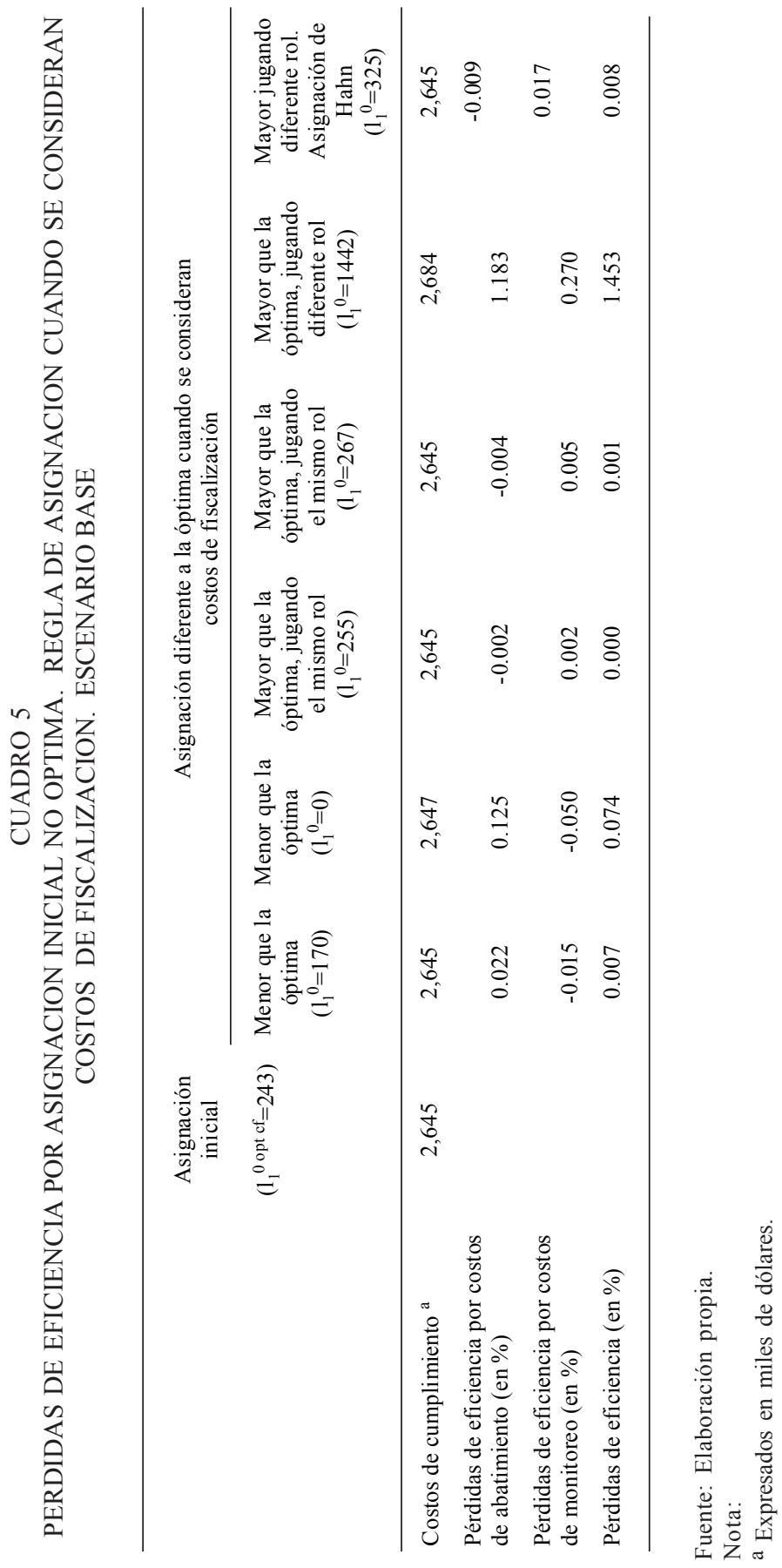


Considerando la reducida magnitud de los impactos de eficiencia ocasionados por asignaciones de permisos a la firma dominante, diferentes de aquélla óptima obtenidos a partir de las simulaciones en el escenario base, decidimos evaluar si dichos resultados son robustos a distintas especificaciones de los parámetros utilizados. Con dicho objetivo, realizamos un análisis de sensibilidad, para lo cual se simularon diferentes escenarios utilizando parámetros alternativos. Los escenarios simulados pueden dividirse en cuatro grupos según el parámetro que fue alterado: i) escenarios con cambios en el objetivo ambiental, ii) escenarios con cambios en la estructura de sanciones y costos de auditoría, iii) escenarios con cambios en el número de fuentes participantes en el mercado y finalmente, iv) un escenario en el que se evalúa la sensibilidad ante cambios en los parámetros de la función de costos marginales de abatimiento de la fuente con poder de mercado. En el Gráfico 1 se presentan los resultados obtenidos respecto de las pérdidas de eficiencia para los escenarios simulados, en dos casos extremos: cuando se asignan todos los permisos a la fuente dominante $\left(1_{1}^{0}=\mathrm{L}\right)$, y cuando no se le entrega ningún permiso a dicha fuente $\left(l_{1}{ }^{0}=0\right) .{ }^{19}$ Se observa allí que la mayor magnitud para la pérdida de eficiencia se produce en el caso en el que se tiene una regulación más estricta, con una meta ambiental de descontaminación mayor y se asignan inicialmente todos los permisos a la fuente con poder de mercado, en este caso el valor para la pérdida de eficiencia es $9,2 \%$.

En el escenario en el cual las sanciones marginales por violación son más severas, puede observarse que los costos totales se reducen y la pérdida de eficiencia sigue siendo leve. Ello implica que la aplicación de una política con sanciones más severas, que asegura cumplimiento, no tendría mayores costos sociales.

Adicionalmente, y con el propósito de complementar el análisis, se realizaron ejercicios de simulación para funcionamiento del sistema de permisos de emisión, suponiendo que la fuente que ejerce poder de mercado posee un porcentaje en el nivel de emisiones iniciales mayor a $11 \%$. Se simularon los casos en los cuales el nivel de emisiones iniciales de dicha fuente es $20 \%, 30 \%$, y $50 \% .{ }^{20}$ Los resultados obtenidos con base en dichas simulaciones son reportados en el Cuadro 6. 
GRAFICO 1

PERDIDAS DE EFICIENCIA EN LOS ESCENARIOS

SIMULADOS - ANALISIS DE SENSIBILIDAD

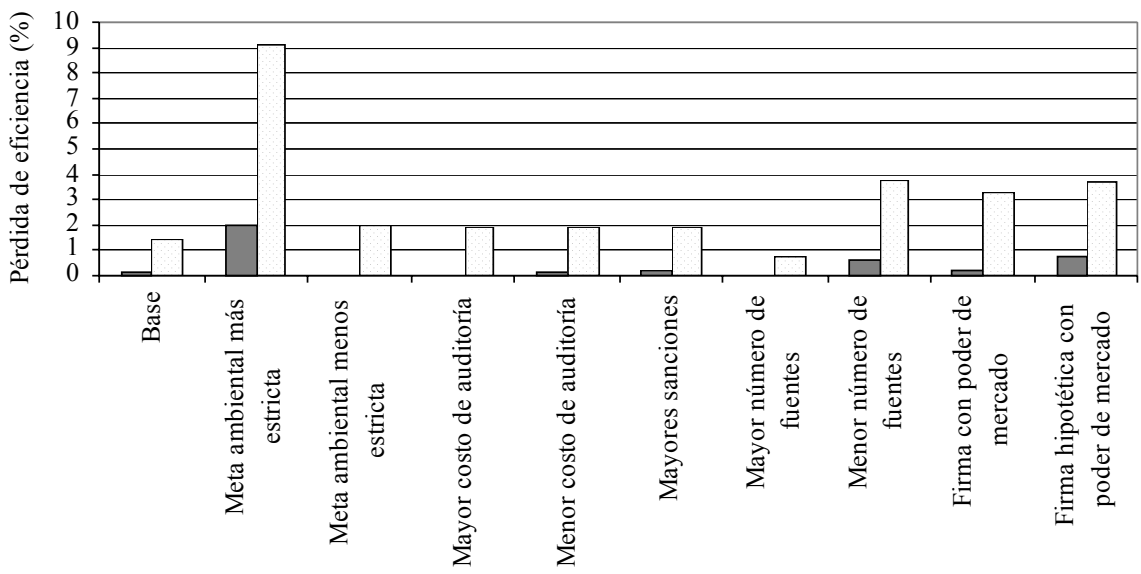

Inicialmente ningún permiso a fuente dominante

$\square$ Inicialmente to dos los permisos a fuente dominante

CUADRO 6

PERDIDAS DE EFICIENCIA POR ASIGNACION INICIAL NO OPTIMA

PARA DIFERENTES PORCENTAJES DE EMISIONES INICIALES DE LA FUENTE CON PODER DE MERCADO

\begin{tabular}{|c|c|c|c|c|c|c|c|c|c|c|}
\hline \multirow[b]{2}{*}{$\begin{array}{c}\text { Porcentaje } \\
\text { de } \\
\text { participación }\end{array}$} & \multicolumn{4}{|c|}{ Asignación diferente a Regla de Hahn } & \multicolumn{6}{|c|}{ Asignación diferente a la de Chávez y Stranlund } \\
\hline & $\begin{array}{c}\text { Mayor que } \\
\text { la óptima } \\
\text { en } 30 \%\end{array}$ & $\begin{array}{l}\text { Menor que } \\
\text { la óptima } \\
\text { en 30\% }\end{array}$ & $\begin{array}{c}\text { Todos los } \\
\text { permisos }\end{array}$ & $\begin{array}{l}\text { Ningún } \\
\text { permiso }\end{array}$ & $\begin{array}{r}\mathrm{Me} \\
\text { optim } \\
\text { el } \mathrm{m}\end{array}$ & $\begin{array}{l}\text { ra la } \\
\text { jugando } \\
\text { no rol }\end{array}$ & $\begin{array}{r}\mathrm{M} \\
\text { jug } \\
\text { el mi }\end{array}$ & $\begin{array}{l}\text { or } \\
\text { hdo } \\
\text { no rol }\end{array}$ & $\begin{array}{l}\text { Mayor } \\
\text { jugando } \\
\text { diferente }\end{array}$ & $\begin{array}{c}\text { Asignación } \\
\text { Hahn }\end{array}$ \\
\hline 11 & 0.01 & 0.01 & 1.38 & 0.15 & 0.01 & 0.07 & 0 & 0 & 1.45 & 0.01 \\
\hline 20 & 2.27 & 0.10 & 4.07 & 1.04 & 0.08 & 0.78 & 0.01 & 0.02 & 2.47 & 0.01 \\
\hline 30 & 2.72 & 0.42 & 4.72 & 17.89 & 0.51 & 17.91 & 0.13 & 0.14 & 13.17 & 13.81 \\
\hline 50 & 1.12 & 14.45 & 1.12 & 100.68 & 13.93 & 97.26 & 0.58 & 0.63 & 0.63 & 0.63 \\
\hline
\end{tabular}

Fuente: Elaboración propia.

Las cifras de la tabla representan las pérdidas de eficiencia, y están expresadas en porcentaje.

a Expresada en porcentaje. Representa el porcentaje de participación de la fuente dominante en el nivel de emisiones iniciales totales.

b. Asignación extrema. Se asignan inicialmente todos los permisos a la fuente dominante.

c. Asignación extrema. No se asigna inicialmente ningún permiso a la fuente dominante.

d. Asignación extrema. Se asignan inicialmente todos los permisos a la fuente dominante. 
Nuestros resultados indican ausencia de efectos significativos en términos de eficiencia cuando la fuente dominante mantiene niveles de participación en las emisiones iniciales inferiores a 20\%. Sin embargo, los resultados cambian, para las dos reglas de asignación, en los casos en los cuales las simulaciones suponen que la fuente dominante posee el $30 \%$ y $50 \%$. En efecto, a medida que aumenta el porcentaje de emisiones iniciales que tiene la fuente que ejerce poder de mercado, esta pérdida de eficiencia puede hacerse significativa. Específicamente, esta pérdida de eficiencia tiene un valor, para el caso en el cual, siguiendo la regla de Hahn (1984), se hacen asignaciones iniciales diferentes a la óptima; sobre todo para aquellas asignaciones extremas, esto es, cuando no se asigna ningún permiso a la fuente que ejerce poder de mercado, o cuando se asignan todos los permisos a la misma. Para el caso en el cual se estudia la asignación siguiendo la regla de Chávez y Stranlund (2003), las pérdidas de eficiencia se hacen importantes para el caso en el que no se concede ningún permiso a la firma con poder de mercado.

\section{CONCLUSIONES}

En este artículo estudiamos el funcionamiento de un sistema de permisos de emisión transferibles en un contexto en el cual, además de considerar la existencia de poder de mercado, se tienen en cuenta los costos de fiscalización para inducir cumplimiento de la regulación.

El trabajo empírico se basó principalmente en el modelo teórico sobre diseño de fiscalización de un SPET en presencia de poder de mercado de Chávez y Stranlund (2003). El modelo teórico desarrollado por dichos autores se aplicó al caso del Programa de Compensación de Emisiones (PCE) de la ciudad de Santiago de Chile en el año de 1997. A través de simulaciones estáticas, se cuantificaron los costos de cumplimiento y la pérdida de eficiencia generada por asignaciones iniciales diferentes a la óptima determinada en este caso.

En la estimación de la pérdida de eficiencia, se utilizaron funciones de costo marginal de abatimiento derivadas de las funciones de costo marginal de abatimiento anual agregado presentadas por Montero et al. (2002) a partir de un procedimiento que incorpora un factor de corrección cuyo valor depende de características específicas de las fuentes, y cuyo objetivo es captar la heterogeneidad en los costos marginales de abatimiento existentes entre las fuentes. La escasez de información acerca de las funciones de costo marginal de abatimiento de cada fuente, y la necesidad de recurrir a un procedimiento alternativo para obtener estas funciones, constituye una limitación del presente trabajo.

La pérdida de eficiencia ocasionada por diferentes asignaciones iniciales de permisos es medida a través de la diferencia en los costos totales de la regulación cuando se hace una asignación inicial óptima y una diferente de la óptima. Dado que esa pérdida de eficiencia depende de los niveles de costos, previo al cálculo de su magnitud, se estudió la variación de los costos de cumplimiento para diferentes niveles de abatimiento cuando se considera que existe competencia perfecta, cuando se tiene en cuenta sólo el poder de mercado de acuerdo a Hahn 
(1984) y cuando se incluyen en el análisis además del poder de mercado, los costos de fiscalización, de acuerdo a lo sugerido por Chávez y Stranlund (2003).

Del análisis de estos niveles de costos, cuando se estudian asignaciones iniciales diferentes a la óptima, se concluye que en el caso en el cual se considera competencia perfecta, los costos totales del programa son subestimados con respecto al caso en el cual se tienen en cuenta los costos de fiscalización y emisión de reportes en una magnitud que fluctúa entre US\$ 958 mil para un nivel de abatimiento de $10 \%$ y US\$ 1.20 millones para un nivel de abatimiento de $90 \%$.

La diferencia entre los niveles de costos para los casos en los que se considera que el poder de mercado y competencia perfecta no son muy amplias. Esto se debe a que las transacciones de la fuente con poder de mercado no afectan de manera significativa el precio de los permisos y, por tanto, tampoco las decisiones de niveles de emisiones por parte de las fuentes restantes. Lo anterior se ve finalmente reflejado en la reducida variación de los costos totales del programa, lo que sugiere que para el caso del PCE, el impacto del ejercicio de poder de mercado sobre los costos de cumplimiento es bajo. Nuestros resultados sugieren que, a pesar del posible ejercicio de poder de mercado en el contexto de un mercado de permisos de emisión, un SPET puede ser incluso superior a otros instrumentos de regulación ambiental en términos de su costo-efectividad.

Es importante señalar en este punto que para el estudio de los niveles de costos, en el caso en el cual se tienen en cuenta los costos de fiscalización, se consideraron además los costos de reporte de emisiones. Los costos de reportar las emisiones se suponen independientes del nivel de abatimiento y de la asignación inicial de permisos a la fuente con poder de mercado.

Una vez estudiados los niveles de costos, se calculó la pérdida de eficiencia ocasionada por asignaciones iniciales diferentes a la óptima. En el caso en el que se asignó a la fuente con poder de mercado una cantidad de permisos mayor o menor que la óptima, se incurre en una pérdida de eficiencia. Esta pérdida aumenta cuando crece la diferencia entre la asignación inicial óptima y la asignación inicial realizada, siendo máxima en el caso extremo en el que se asignan todos los permisos a la fuente dominante.

La magnitud de la pérdida de eficiencia encontrada en las simulaciones es baja y esto puede deberse al bajo impacto del ejercicio de poder de mercado. Al observar las variaciones en el precio de mercado de los permisos, estos experimentan cambios muy pequeños cuando la asignación inicial es diferente a la óptima.

El resultado anterior puede estar en parte determinado con el criterio utilizado para elegir a la fuente que ejerce poder de mercado. La forma idónea para determinar cuál fuente es la que ejerce este poder, es observar información referente a las transacciones y al precio de los permisos: si la transacción de una fuente induce un cambio significativo en el precio de los permisos, ésta puede considerarse como la que tiene poder de mercado; pero dado que el mercado en el PCE de Santiago ha sido muy poco desarrollado, se consideró que la fuente con una mayor concentración de emisiones y por tanto de permisos, es la que ejerce poder de mercado. Naturalmente, esto podría constituirse una limitación adicional del trabajo. 
Considerando los resultados encontrados, puede concluirse que los efectos potenciales de poder de mercado no parecen ser muy relevantes para el caso de estudio. La distribución inicial de permisos no afecta en mayor medida el funcionamiento del mercado; ello ocurre incluso en situaciones en que la distribución de permisos que recibe la empresa dominante difiere significativamente de aquella que minimiza los costos totales del programa.

No obstante, aunque la pérdida de eficiencia encontrada es muy baja, no es posible hacer una generalización y restarle relevancia a la asignación inicial de permisos cuando se consideran costos de fiscalización y poder de mercado. Este comportamiento puede deberse a condiciones específicas del caso estudiado.

$\mathrm{Al}$ realizar un análisis de sensibilidad de la magnitud de la pérdida de eficiencia se observa que ésta es más sensible a cambios en el parámetro referido a la meta ambiental. Específicamente, cuando la meta de reducción de emisiones es menor, la magnitud de la pérdida de eficiencia disminuye. Cuando el objetivo de reducción se hace más estricto la magnitud de la pérdida de eficiencia se incrementa.

Un resultado adicional importante que surge del análisis de sensibilidad es aquel observado cuando las sanciones marginales por violación son más severas. En dicho caso, notamos que los costos totales se reducen y la pérdida de eficiencia continúa siendo leve. Ello sugiere que la aplicación de una política con sanciones más severas que asegura cumplimiento no tendría mayores costos en términos de pérdida de eficiencia.

Los resultados obtenidos a partir de la realización de simulaciones como las presentadas en este trabajo pueden ser útiles en el contexto de diseño de una regulación ambiental basada en un SPET: si la pérdida de eficiencia obtenida por asignaciones iniciales diferentes a la óptima es de una magnitud importante, puede constituirse en un llamado de atención a la autoridad para que en el diseño del mercado tenga especial atención sobre el potencial ejercicio del poder de mercado y sobre la asignación inicial de permisos a las fuentes participantes en el mercado. En caso contrario, y tal como sugieren estos resultados, el diseñador de política dispone de grados de libertad mayores para concentrarse en cuestiones quizás relacionadas a aceptabilidad política u otras consideraciones, al decidir respecto de la asignación inicial de permisos.

Finalmente, cabe discutir la posibilidad de estudiar en trabajos futuros la pérdida de eficiencia ocasionada por asignaciones iniciales diferentes a la óptima, en un contexto en el que se consideran los costos de fiscalización para inducir cumplimiento de la regulación, en presencia de una estructura oligopólica, es decir; cuando el mercado de permisos está caracterizado por la presencia de varias firmas que tienen la posibilidad de ejercer poder de mercado, en lugar de una sola de ellas, como se plantea en este trabajo. Igualmente, puede ser importante, como área futura de estudio la pregunta referida a cuál es el nivel de cumplimiento apropiado dados los costos de las inspecciones, pregunta que en nuestro conocimiento, no está ni abordada ni resuelta en la literatura relacionada. 


\section{REFERENCIAS}

Chávez, C. A, y J. K. Stranlund (2003), "Enforcing Transferable Permit Systems in the Presence of Market Power", Environmental \& Resource Economics, 25: 65-78.

Hahn, R. (1984), "Market power and transferable property rights", Quarterly Journal of Economics, 99: 735-765.

Innes, R.; C. Kling, y J. Rubin (1991), "Emission Permits Under Monopoly”, Natural Resource Modelling, 5 (3): 321-343.

Malik, A. S. (2002), "Further Results on Permit Markets with Market Power and Cheating", Journal of Environmental Economics and Management, 44 (3): 371-390.

Misiolek, W. y H. W. Elder (1989), "Exclusionary Manipulation of Markets for Pollution Rights". Journal of Environmental Economics and Management 16: 156-166.

Montero, J.P.; J.M., Sánchez; R. Katz (2001), “Análisis del Mercado de Emisiones de Material Particulado en Santiago”, Estudios Públicos 81: 97-126.

Montero, J.P., J.M. Sánchez y R. Katz (2002), “A Market-Based Environmental Policy Experiment in Chile", Journal of Law and Economics, 45 (1): 267-287.

Montgomery, W. (1972), "Markets in Licenses and Efficient Pollution Control Programs", Journal of Economic Theory, 5: 395-418.

Palacios, M. y C. Chávez (2002), "Programa de Compensación de Emisiones: Evaluación del Diseño de Fiscalización y su Cumplimiento", Estudios Públicos, 88: $97-126$.

Van Egteren, H. y M. Weber (1996), "Marketable Permits, Market Power, and Cheating", Journal of Environmental Economics and Management, 30: 161-173.

Villena, M. y M. Villena (1998), “Analyzing Economic Strategies for Air Pollution control in Santiago, Chile", Environmental Series, Number 4, Department of Land Economy, University of Cambridge.

Westskog, H. (1996), "Market Power in a System of Tradeable $\mathrm{CO}_{2}$ Quotas", The Energy Journal 17 (3): 85-103. 
ANEXO 1

DESCRIPCION DE PROCESOS TIPO

\section{CUADRO A-1 \\ CARACTERIZACION PROCESOS TIPO}

\begin{tabular}{lclc}
\hline Proceso utilizado & $\begin{array}{c}\text { Número de } \\
\text { fuentes }\end{array}$ & $\begin{array}{l}\text { Combustible } \\
\text { utilizado }\end{array}$ & $\begin{array}{c}\text { Número de } \\
\text { fuentes }\end{array}$ \\
\hline Caldera de calefacción & 14 & Aserrín & 3 \\
Caldera de calefacción y agua caliente & 4 & Carbón bituminoso & 3 \\
Caldera agua caliente & 1 & Gas de cañería & 2 \\
Caldera de fluido térmico & 2 & Gas licuado & 6 \\
Caldera industrial & 15 & Kerosene & 5 \\
Caldera generadora de vapor & 12 & Lef & 4 \\
Generador de vapor & 30 & Leña & 4 \\
Caldera superior & 1 & LMFO & 5 \\
Caldera gas & 1 & Petróleo & 34 \\
& & Skygard & 4 \\
& & Superlef & 7 \\
& & Viruta & 1 \\
& & Gas Natural & 1 \\
& & Chil & 1 \\
\hline
\end{tabular}

Fuente: Elaboración propia a partir de base de datos proporcionados por el SESMA.

Notas:

a número de fuentes que tienen cada tipo de proceso

${ }^{\mathrm{b}}$ número de fuentes que utilizan cada tipo de combustible. 


\section{ANEXO 2 \\ OBTENCION DE FUNCIONES BASE DE COSTO MARGINAL DEABATIMIENTO}

Para fuentes existentes, la función agregada de costos marginales de abatimiento de acuerdo con Montero et al. (2002) es:

$$
\mathrm{CMA}_{\mathrm{E}}=360+1.05 * \mathrm{Q}
$$

donde Q son las reducciones (kg/día). Despejando las reducciones, se obtiene:

$$
\mathrm{Q}=\frac{\mathrm{CMA}_{\mathrm{E}}-360}{1.05}
$$

Si se considera que las reducciones totales son el producto entre el número de fuentes existentes y las reducciones de cada una:

$$
\begin{aligned}
& \mathrm{Q}=\mathrm{n}_{\mathrm{E}} *\left(\mathrm{q}_{\mathrm{i}}^{\mathrm{E}}\right) \\
& \mathrm{n}_{\mathrm{E}} * \mathrm{q}_{\mathrm{i}}^{\mathrm{E}}=\frac{\mathrm{CMA}_{\mathrm{E}}}{1.05}-\frac{360}{1.05}
\end{aligned}
$$

Donde :

$\mathrm{n}_{\mathrm{E}}$ : número de fuentes existentes.

$\mathrm{q}_{\mathrm{i}} \mathrm{E}$ : reducción de la fuente existente $\mathrm{i}$.

$$
\mathrm{q}_{\mathrm{i}}^{\mathrm{E}}=\frac{\mathrm{CMb}_{\mathrm{E}}}{1.05 \times \mathrm{n}_{\mathrm{E}}}-\frac{360}{1.05 \times \mathrm{n}_{\mathrm{E}}}
$$

Despejando los costos marginales de abatimiento se obtiene la función base para los mismos:

$$
\mathrm{CMb}_{\mathrm{E}}=360+1.05 \times \mathrm{n}_{\mathrm{E}} \times \mathrm{q}_{\mathrm{i}}^{\mathrm{E}}
$$

Siguiendo el mismo procedimiento para fuentes nuevas, se obtiene la función de costos marginales de abatimiento base.

La función agregada de costos marginales de abatimiento presentada por Montero et al. (2002) es:

$\mathrm{CMA}_{\mathrm{N}}=23.73 * \mathrm{Q}$ donde $\mathrm{Q}$ son las reducciones (kg/día).

Despejando las reducciones, se obtiene:

$\mathrm{Q}=\frac{\mathrm{CMA}_{\mathrm{N}}}{23.73}$ 


\section{Suponiendo $Q=\mathrm{n}_{\mathrm{N}} * \mathrm{q}_{\mathrm{i}}^{\mathrm{N}}$}

con $\mathrm{q}_{\mathrm{i}}^{\mathrm{N}}$ reducciones de la firma nueva $\mathrm{i}$.

$$
\begin{aligned}
& \mathrm{n}_{\mathrm{N}} \mathrm{q}_{\mathrm{i}}^{\mathrm{N}}=\frac{\mathrm{CMb}_{\mathrm{N}}}{23.73} \\
& \mathrm{q}_{\mathrm{i}}^{\mathrm{N}}=\frac{\mathrm{CMb}_{\mathrm{N}}}{\mathrm{n}_{\mathrm{N}} * 23.73} \\
& \mathrm{CMb}_{\mathrm{N}}=23.73 *\left(\mathrm{e}_{\mathrm{iN}}^{0}-\mathrm{e}_{\mathrm{iN}}\right)
\end{aligned}
$$

Las fuentes competitivas eligen sus emisiones en un nivel tal que sus costos marginales igualen el precio

$$
\begin{aligned}
& \operatorname{CMg}_{2}\left(\mathrm{e}_{2}\right)=\mathrm{a}_{2} \mathrm{e}_{2}+\mathrm{b}_{2}=\mathrm{p} \\
& \mathrm{CMg}_{3}\left(\mathrm{e}_{3}\right)=\mathrm{a}_{3} \mathrm{e}_{3}+\mathrm{b}_{3}=\mathrm{p} \\
& \mathrm{CMg}_{\mathrm{n}}\left(\mathrm{e}_{\mathrm{n}}\right)=\mathrm{a}_{\mathrm{n}} \mathrm{e}_{\mathrm{n}}+\mathrm{b}_{\mathrm{n}}=\mathrm{p}
\end{aligned} \quad \text { con } a_{i}<0 \text { y } b_{i}>0
$$

Si se despeja el nivel de emisiones de cada una de estas ecuaciones, se obtiene:

$$
\begin{aligned}
& e_{2}=\frac{p-b_{2}}{a_{2}} \\
& e_{3}=\frac{p-b_{3}}{a_{3}} \\
& e_{n}=\frac{p-b_{n}}{a_{n}}
\end{aligned}
$$

La demanda de permisos por cada una de las fuentes será igual a su nivel de emisiones, si se suma entonces las emisiones para todas las fuentes competitivas que demandan permisos, se obtiene la demanda de permisos por parte de las firmas. $^{21}$ 


$$
\sum_{i=2}^{n} e_{i}(p)=p * \sum_{i=2}^{n} \frac{1}{a_{i}}-\sum_{i=2}^{n} \frac{b_{i}}{a_{i}}
$$

Donde i es el número de firmas que demandan permisos. Definimos:

$$
\mathrm{k}=\sum_{\mathrm{i}=2}^{\mathrm{n}} \frac{1}{\mathrm{a}_{\mathrm{i}}}, \mathrm{d}=-\sum_{\mathrm{i}=2}^{\mathrm{n}} \frac{\mathrm{b}_{\mathrm{i}}}{\mathrm{a}_{\mathrm{i}}}
$$

Entonces:

$$
\sum_{i=2}^{n} e_{i}(p)=d+k * p
$$

La anterior es la función de demanda por permisos por parte de las fuentes competitivas.

Dado que para algunos precios de equilibrio algunas fuentes eligen un nivel de emisiones de cero, es decir, no demandan permisos, la función de demanda se presenta como una función de demanda por tramos. 